\title{
ESTRÉS LABORAL EN EL PERSONAL DE ENFERMERÍA DEL ÁREA DE UCI DURANTE LA PANDEMIA DE COVID 19
}

\author{
AUTORES: María Tatiana Betancourt Delgado ${ }^{1}$ \\ Wilson Fabricio Domínguez Quijije ${ }^{2}$ \\ Brandon Israel Peláez Flores ${ }^{3}$ \\ María del Rosario Herrera Velázquez ${ }^{4}$
}



DIRECCIÓN PARA CORRESPONDENCIA: (maria8468@unesum.edu.ec)

Fecha de recepción: 2/09/2020

Fecha de aceptación: 11/11/2020

\section{RESUMEN}

Objetivo: Determinar el nivel de estrés de los licenciados en enfermería y auxiliares de enfermería e internos de enfermería durante la Pandemia de Covid 19. Metodología: Se realizó un estudio observacional, analítico, de corte transversal en el mes de agosto de 2020 en el hospital Rodríguez Zambrano de Manta en el área de Unidad de Cuidados Intensivos (UCI). Se incluyó a todos los licenciados en enfermería y auxiliares de enfermería e internos de enfermería del Hospital Rodríguez Zambrano, que laboran en el área de cuidados intensivos que actualmente debido a la emergencia sanitaria laboran durante 13 horas diarias. La encuesta aplicada es "The Nursing Stress Scale”. La adaptación cultural de la escala se hizo por medio del método de traducción-retrotraducción, cuyos autores son V. Escribá/ R. Más/ M. Cárdenas/ S. Pérez. Publicada en la página de sciencedirect.com. Resultados: Participaron en el estudio 14 profesionales de enfermería, 2 auxiliares de enfermería y 8 internos de enfermería, con edad promedio de 21 a 40 años de edad, se obtuvo como resultado que factores de muerte y sufrimiento, carga laboral son los causantes de mayor índice de estrés laboral. Conclusión: En la investigación, está claro que existe mayor índice de estrés laboral en el género femenino y es indispensable evaluar los factores estresantes en función de los mecanismos de afrontamiento disponibles, a fin de minimizar la probabilidad de que las personas experimenten estrés.

PALABRAS CLAVE: estrés, enfermería, ambiente laboral, pandemia.

\section{WORK STRESS IN NURSING STAFF IN THE ICU AREA DURING THE COVID 19 PANDEMIC}

\section{ABSTRACT}

\footnotetext{
${ }^{1}$ Internos de Enfermería, octavo semestre, Universidad Estatal del Sur de Manabí, Ecuador. E-mail: maria8468@unesum.edu.ec

${ }^{2}$ Internos de Enfermería, octavo semestre, Universidad Estatal del Sur de Manabí, Ecuador.

${ }^{3}$ Internos de Enfermería, octavo semestre, Universidad Estatal del Sur de Manabí, Ecuador.

${ }^{4}$ Universidad Estatal del Sur de Manabí, Ecuador.
} 
Objective: To determine the stress level of nursing graduates and nursing assistants and nursing interns during the Covid 19 Pandemic. Methodology: An observational, analytical, crosssectional study was carried out in August 2020 at the hospital Rodríguez Zambrano de Manta in the area of the Intensive Care Unit (ICU). All nursing graduates and nursing assistants and nursing interns from Hospital Rodríguez Zambrano, who work in the intensive care area, who currently work 13 hours a day due to the health emergency were included. The survey applied is "The Nursing Stress Scale" The cultural adaptation of the scale was done by means of the translation-back-translation method, whose authors are V. Escribá / R. Más / M. Cárdenas / S. Pérez. Posted on the sciencedirect.com page. Results: 14 nursing professionals, 2 nursing assistants and 8 nursing interns participated in the study, with an average age of 21 to 40 years old, it was obtained as a result that factors of death and suffering, workload are the causes of greater work stress index. Conclusion: In the research, it is clear that there is a higher rate of work stress in the female gender and it is essential to evaluate the stressors based on the available coping mechanisms, in order to minimize the probability that people experience stress.

KEYWORDS: stress, nursing, work environment, pandemic.

\section{INTRODUCCIÓN}

El estrés es un fenómeno que ha acompañado a la humanidad desde sus orígenes de tal forma que personajes como el filósofo Hipócrates hacía referencia a este cuando hablaba de adversidad, aflicción, experiencias negativas y sufrimiento (Félix Verduzco, García Hernández, \& Mercado Ibarra, 2018).

El estrés es un fenómeno más común en nuestra sociedad, porque está constantemente nos enfrentamos a nuevos retos o retos en la vida profesional y personal. En el mundo laboral, responsabilidades, cambios constantes, decisiones difíciles, nuevas exigencias profesionales, etc. Se pueden convertir a muchas fuentes de estrés para el profesional en la actualidad. Por eso, aprender a conocer las señales de estrés y crear estrategias específicas cada vez más importantes para erradicarlo en las organizaciones y los empleados (Vargas García \& Garcia Alcivar, 2018).

El estrés es la respuesta física, psicológica y de conducta de las personas que intenta adaptarse y ajustarse a las presiones internas y externas. Cuando las personas, el trabajo y la organización no coinciden, se produce una presión laboral (Aray Mendoza \& Acosta Andrade, 2016). Por otro lado, la Organización Mundial de la Salud (OMS) ha determinado que el estrés es un conjunto de respuestas fisiológicas que preparan al organismo para la acción. La organización Internacional del Trabajo (OIT) se refieres al estrés laboral en los siguientes términos; esta enfermedad representa una amenaza para las economías de los países industriales y en desarrollo. Al afectar la salud física y mental de los trabajadores, odia la productividad (Chiang Vega, Riquelme Neira, \& Rivas Escobar, 2018).

El estrés se puede definir como la respuesta del cuerpo a necesidades específicas. El endocrinólogo canadiense Hans Selye estudió estas reacciones y, además de los síntomas específicos de la enfermedad de cada persona, sus pacientes también mostraron síntomas sistémicos similares, como fatiga, pérdida de apetito, agotamiento y fiebre (Ospina Stepanian, 2016).

El estrés laboral puede provocar absentismo, baja productividad, alta tasa de rotación, enfermedades y accidentes laborales, etc. Entre el 50\% y el $60 \%$ del ausentismo está relacionado

42 UNESUM-Ciencias. Publicación cuatrimestral. Vol. 4, No. 3 (Número Especial), Año 2020. 
con la presión laboral. Diferentes encuestas realizadas por un estudio en Colombia, muestran la relación entre el desempeño del estrés laboral y diversos aspectos, como el tipo de servicio que brinda la organización, la carga mental, la duración de la jornada laboral y la frustración con las tareas (Sarsosa Prowesk \& Charria Ortiz, 2018).

Un estudio realizado en el Ecuador por la GFK (Gesellschaft für Konsumforschung encuestadora alemana) determinó que el $10 \%$ de los ecuatorianos sufren de estrés laboral y el 14\% tiene el colesterol alto. Esta investigación se realizó en las principales ciudades, como son Guayaquil y Quito, aproximadamente alrededor de 1006 personas, 514 en el puerto principal y 492 en la ciudad capital. Por otro lado, se evidencio que el estrés laboral está asociado a diferentes enfermedades, como la gastritis, que tiene el $8 \%$, cifra muy cercana al índice de estrés laboral (Yunda Ibarra, 2018).

El clima laboral negativo y el estrés van de la mano, porque cuando se trata de clima organizacional, el clima laboral en que las personas interactúan dentro de la organización afecta su compromiso. La presión laboral es un resultado negativo de la interacción de los empleados. Ante los cambios de globalización, se debe considerar la capacidad de respuestas de los empleados. Un buen clima organizacional tendrá consecuencias positivas, tales como: logro, afiliación, satisfacción, adaptabilidad, innovación y otros aspectos, que ayudaran a la organización a alcanzar sus metas (Vicente \& Yesenia, 2017).

Los profesionales de enfermería están sometidos a mucha presión por el entorno en el que desarrollan su trabajo, la consideran una ocupación muy estresante y tiene una alta prevalencia de enfermedades mentales relacionadas con el estrés, la insatisfacción laboral, el absentismo y los frecuentes cambios de trabajo. La presión laboral es uno de los motivos más importante en esta situación, ya que tiene las características de manejo de situaciones de emergencia y ejecución eficiente, por los que muchos autores señalan que esta profesión requiere enormes responsabilidades y por ende a presiones, conflictos y cambios constantes (Reyes \& Milagros, 2019).

El trabajo del enfermero insertado en las instituciones de salud, tiende a estar sometido a soportar diversas cargas y causar desgaste. Por otro lado, el trabajo también es una fuente de placer y satisfacción, mejorando la capacidad del ser humano para promover la salud y la vida (Lopes Guerrer \& Ferraz Bianchi, 2015).

La presión laboral en una epidemia mundial afecta la salud física y mental al equipo de salud, especialmente de enfermería. Según Cremades, el estrés ocurrirá en la sala de emergencias, unidad de cuidados intensivos y en quirófano. Debido a ciertos factores de estrés, como el exceso de trabajo significa alto sentido de responsabilidad, falta de personal y de tiempo para terminar las tareas de enfermería. Los individuos se sentirán cansados y provocarán rendimiento deficiente, desempeño laboral, ausentismo y abandono de la carrera (Medina Espinoza, 2017).

Por lo tanto, el personal de enfermería puede obtener fácilmente una enfermedad fisiológica, física y mental; poniendo en peligro su vida. La Organización mundial de la salud, dispone que para el año 2020 la depresión por estrés será la segunda causa de morbilidad en relación a enfermedades cardiovasculares, endocrinas que llevaran a la jubilación temprana y aumento de los costos médicos de los enfermeros (Medina Espinoza, 2017). 
María T. Betancourt, Wilson F. Domínguez, Brandon I. Peláez, María del Rosario Herrera.

\section{DESARROLLO}

Se realizó un estudio observacional, analítico, de corte transversal en el mes de agosto de 2020 en el hospital Rodríguez Zambranode Manta en el área de Unidad de Cuidados Intensivos (UCI).

\section{Población}

Se incluyó a todos los licenciados en enfermería y auxiliares de enfermería e internos de enfermería del Hospital Rodríguez Zambrano, que laboran en el área de cuidados intensivos que actualmente debido a la emergencia sanitaria laboran durante 13 horas diarias.

Se encuesta al personal voluntario a participar en llenar la encuesta con la ayuda de los miembros del equipo que estuvieron presentes para obtener los datos pertinentes en el llenado de las encuestas, posteriores a valorar según las escalas del estudio.

Instrumento

La encuesta aplicada es “The Nursing Stress Scale” La adaptación cultural de la escala se hizo por medio del método de tradución-retrotraducción, cuyos autores son V. Escribá/ R. Más/ M. Cárdenas/ S.Pérez. Publicada en la página de sciencedirect.com.

La encuesta se aplica de manera física, fue anónima y consta de 34 ítems, Estos ítems han sido agrupados en siete factores; uno de ellos relacionado con el ambiente físico (carga de trabajo), cuatro con el ambiente psicológico (muerte y sufrimiento, preparación insuficiente, falta de apoyo e incertidumbre en el tratamiento) y dos relacionados con el ambiente social en el hospital (problemas con los médicos y problemas con otros miembros del equipo de enfermería). En cada ítem las posibles respuestas son: nunca (0), a veces (1), frecuentemente (2) y muy frecuentemente (3). Sumando las puntuaciones obtenidas en cada uno de ellos se obtiene un índice global cuyo rango se encuentra entre 0 y 102, de forma que a mayor puntuación mayor nivel de estresores. Se trata de una escala auto administrada cuya cumplimentación se realiza aproximadamente en 10 minutos.

\section{RESULTADOS}

Participaron en el estudio 14 profesionales de enfermería, 2 auxiliares de enfermería y 8 internos de enfermería, con edad promedio de 21 a 40 años de edad); de estos, 83\% (20) son mujeres y el $17 \%$ (4) son hombres. En cuanto al grado de escolaridad el 67\% (16) tiene un título de tercer Nivel y el 33\% (8) son profesionales en formación (Internos de Enfermería).

\section{Características laborales de las enfermeras}

Al determinar la vinculación laboral de los trabajadores estudiados, se encontró que el tipo de contratación más común es a término temporal 79\% (19).

El 100\% (24) de los participantes labora en el área de Unidad de Cuidado Intensivos. Los cuales realizan turnos rotativos, en promedio por turnos atienden a 8 pacientes. Al indagar sobre la realización de actividades extra laborales, la mayoría realiza actividades como ama casa y compartiendo con sus familiares.

Nivel de estrés de los participantes en el estudio

44 UNESUM-Ciencias. Publicación cuatrimestral. Vol. 4, No. 3 (Número Especial), Año 2020. 
Publicación cuatrimestral. Vol. 4, No. 3 (Número Especial), Año 2020. Pág. 41-50 ESTRÉS LABORAL DURANTE LA PANDEMIA DE COVID 19

Al determinar el nivel de estrés laboral de los participantes que laboran en el área de UCI, se obtuvo como resultado que el $21 \%$ de los encuestados está expuesto a un alto nivel de estrés, mientras que el 79\% están expuestos a un bajo nivel de estrés laboral (ver tabla 1).

Tabla 1: Medición del estrés.

\begin{tabular}{|c|c|c|c|c|}
\hline \multicolumn{5}{|c|}{ Resultados obtenidos luego de la Aplicación de la Escala “The Nursing Stress Scale” } \\
\hline & Nunca & $\begin{array}{l}\text { Alguna } \\
\text { vez }\end{array}$ & Frecuentemente & $\begin{array}{c}\text { Muy } \\
\text { frecuentemente }\end{array}$ \\
\hline $\begin{array}{l}\text { 1.-Interrupciones frecuentes en la realización de sus } \\
\text { tareas }\end{array}$ & 4 & 5 & 12 & 3 \\
\hline 2.-Recibir críticas de un médico & 2 & 9 & 8 & 5 \\
\hline $\begin{array}{l}\text { 3.-Realización de cuidados de enfermería que } \\
\text { resultan dolorosos a los pacientes }\end{array}$ & 12 & 2 & 6 & 4 \\
\hline $\begin{array}{l}\text { 4.-Sentirse impotente en el caso de un paciente que } \\
\text { no mejora }\end{array}$ & 20 & 4 & $\mathbf{0}$ & $\mathbf{0}$ \\
\hline 5.-Problemas con un supervisor & 18 & 3 & 2 & 1 \\
\hline $\begin{array}{l}\text { 6.-Escuchar o hablar con un paciente sobre su muerte } \\
\text { cercana }\end{array}$ & 3 & 6 & 11 & 4 \\
\hline $\begin{array}{l}\text { 7.-No tener ocasión para hablar abiertamente con } \\
\text { otros compañeros (enfermeras/os y/o auxiliares de } \\
\text { enfermería) del servicio sobre problemas en el } \\
\text { servicio }\end{array}$ & 2 & 15 & 6 & 1 \\
\hline 8.-La muerte de un paciente & $\mathbf{0}$ & 7 & 16 & 1 \\
\hline 9.-Problemas con uno o varios médicos & 12 & 12 & $\mathbf{0}$ & $\mathbf{0}$ \\
\hline $\begin{array}{l}\text { 10.-Miedo a cometer un error en los cuidados de } \\
\text { enfermería de un paciente }\end{array}$ & 16 & 4 & 3 & 1 \\
\hline $\begin{array}{l}\text { 11.-No tener ocasión para compartir experiencias y } \\
\text { sentimientos con otros compañeros (enfermeras/os } \\
\text { y/o auxiliares de enfermería) del servicio }\end{array}$ & 13 & 5 & 5 & 1 \\
\hline $\begin{array}{l}\text { 12.-Muerte de un paciente con quien has llegado a } \\
\text { tener una relación estrecha }\end{array}$ & 6 & 5 & 2 & 11 \\
\hline $\begin{array}{l}\text { 13.-El médico no está presente cuando un paciente se } \\
\text { está muriendo }\end{array}$ & 19 & 5 & $\mathbf{0}$ & $\mathbf{0}$ \\
\hline $\begin{array}{l}\text { 14.-Estar en desacuerdo con el tratamiento de un } \\
\text { paciente }\end{array}$ & 19 & 5 & $\mathbf{0}$ & $\mathbf{0}$ \\
\hline $\begin{array}{l}\text { 15.-Sentirse insuficientemente preparado para ayudar } \\
\text { emocionalmente a la familia del paciente }\end{array}$ & 10 & 5 & 5 & 4 \\
\hline $\begin{array}{l}\text { 16.-No tener ocasión para expresar a otros } \\
\text { compañeros (enfermeras/os y/o auxiliares de } \\
\text { enfermería) del servicio mis sentimientos negativos } \\
\text { hacia los pacientes (Ej.: pacientes conflictivos, } \\
\text { hostilidad, etc.) }\end{array}$ & 18 & 6 & $\mathbf{0}$ & $\mathbf{0}$ \\
\hline $\begin{array}{l}\text { 17.-Recibir información insuficiente del médico } \\
\text { acerca del estado clínico de un paciente }\end{array}$ & 15 & 5 & 3 & 1 \\
\hline $\begin{array}{l}\text { 18.-No disponer de una contestación satisfactoria a } \\
\text { una pregunta hecha por un paciente }\end{array}$ & 15 & 9 & $\mathbf{0}$ & $\mathbf{0}$ \\
\hline $\begin{array}{l}\text { 19.-Tomar una decisión sobre un paciente cuando el } \\
\text { médico no está disponible }\end{array}$ & 10 & 4 & 4 & 6 \\
\hline 20.-Pasar temporalmente a otros servicios con falta & 20 & 4 & $\mathbf{0}$ & $\mathbf{0}$ \\
\hline
\end{tabular}




\begin{tabular}{|c|c|c|c|c|}
\hline de personal & & & & \\
\hline 21.-Ver a un paciente sufrir & 15 & 9 & $\mathbf{0}$ & $\mathbf{0}$ \\
\hline $\begin{array}{l}\text { 22.-Dificultad para trabajar con uno o varios } \\
\text { compañeros (enfermeras/os y/o auxiliares de } \\
\text { enfermería) de otros servicios }\end{array}$ & 16 & 8 & $\mathbf{0}$ & $\mathbf{0}$ \\
\hline $\begin{array}{l}\text { 23.-Sentirse insuficientemente preparado para ayudar } \\
\text { emocionalmente al paciente }\end{array}$ & 16 & 4 & 2 & 2 \\
\hline 24.-Recibir críticas de un supervisor & 15 & 3 & 2 & 4 \\
\hline 25.-Personal y turno imprevisible & 16 & 8 & $\mathbf{0}$ & $\mathbf{0}$ \\
\hline $\begin{array}{l}\text { 26.-El médico prescribe un tratamiento que parece } \\
\text { inapropiado para el paciente }\end{array}$ & 18 & 6 & $\mathbf{0}$ & $\mathbf{0}$ \\
\hline $\begin{array}{l}\text { 27.-Realizar demasiadas tareas que no son de } \\
\text { enfermería (Ej.: tareas administrativas) }\end{array}$ & 8 & 3 & 3 & 10 \\
\hline $\begin{array}{l}\text { 28.-No tener tiempo suficiente para dar apoyo } \\
\text { emocional al paciente }\end{array}$ & 4 & 3 & 2 & 15 \\
\hline $\begin{array}{l}\text { 29.-Dificultad para trabajar con uno o varios } \\
\text { compañeros (enfermeras/os y/o auxiliares de } \\
\text { enfermería) del servicio }\end{array}$ & 20 & 4 & $\mathbf{0}$ & $\mathbf{0}$ \\
\hline $\begin{array}{l}\text { 30.-No tener tiempo suficiente para realizar todas } \\
\text { mis tareas de enfermería }\end{array}$ & 18 & 6 & $\mathbf{0}$ & $\mathbf{0}$ \\
\hline $\begin{array}{l}\text { 31.-El médico no está presente en una urgencia } \\
\text { médica }\end{array}$ & 20 & 3 & 1 & $\mathbf{0}$ \\
\hline $\begin{array}{l}\text { 32.-No saber que se debe decir al paciente o a su } \\
\text { familia sobre su estado clínico y tratamiento }\end{array}$ & 12 & 9 & 2 & 1 \\
\hline $\begin{array}{l}\text { 33.-No saber bien el manejo y funcionamiento de un } \\
\text { equipo especializado }\end{array}$ & 15 & 9 & 0 & $\mathbf{0}$ \\
\hline $\begin{array}{l}\text { 34.-Falta de personal para cubrir adecuadamente el } \\
\text { servicio }\end{array}$ & 18 & 6 & 0 & $\mathbf{0}$ \\
\hline
\end{tabular}

\section{Causas potenciales de estrés}

\section{Carga laboral}

Al tener interrupciones frecuentes en la realización de sus tareas el 42\% (10) presento estrés. Al realizar demasiadas tareas que no son de enfermería se obtuvo como resultado en un 54\% (13) de los participantes obtuvieron estrés.

Una de las situaciones que representa con mayor frecuencia motivo de estrés para el personal de enfermería es no tener tiempo suficiente para realizar todas las tareas de enfermería $71 \%$ (17) participantes.

\section{Muerte y sufrimiento}

El escuchar o hablar con un paciente sobre su muerte cercana es una situación que frecuentemente constituye la causa de estrés en un 63\% (15) de los encuestados. Estar presente en la muerte de un paciente genera estrés en un 71\% (16) de los participantes. Al analizar la presencia de estrés cuando un paciente con quien ha llegado a tener una relación estrecha muere, se encontró que para el 54\% (13) de los encuestados. Al realizar cuidados de enfermería que resultan dolorosos a los pacientes, se logró obtener que el $42 \%$ (10) de los participantes presento estrés.

Preparación insuficiente

46 UNESUM-Ciencias. Publicación cuatrimestral. Vol. 4, No. 3 (Número Especial), Año 2020. 
$\mathrm{Al}$ 38\% (9) de la población encuestada le causa estrés laboral sentirse insuficientemente preparado para ayudar emocionalmente a la familia del paciente. Se obtuvo un bajo porcentaje de estrés laboral $17 \%$ (4) de los participantes en sentirse insuficientemente preparado para ayudar emocionalmente al paciente ha sido causa de estrés alguna vez en su vida laboral.

Falta de apoyo

Al no tener ocasión para hablar abiertamente con otros compañeros (enfermeras y/o auxiliares de enfermería) del servicio sobre problemas en el servicio, se obtuvo como resultado que el 29\% (7) de los encuestados presentaron estrés. No tener ocasión para compartir experiencias y sentimientos con otros compañeros del servicio, alguna vez ha sido causa de estrés para el $25 \%$ (6) de los participantes.

Incertidumbre en el tratamiento

Al recibir información insuficiente del médico acerca del estado clínico de un paciente se identificó como factor de estrés en un bajo porcentaje el cual es de 17\% (4) de los participantes. Situaciones como no contar con un médico en una urgencia se convierte para el 4\% (1) de los participantes; también el hecho de no saber qué se debe decir al paciente o a su familia sobre su estado clínico y tratamiento representa una fuente de estrés laboral para un .13\% (3) de la población estudiada.

Conflicto con médicos

Al recibir críticas de un médico es causa de estrés en el personal deencuestado, se obtuvo en un 54\% (13) de estrés laboral. Para el 17\% (4) de los participantes, el miedo a cometer un error en los cuidados de enfermería de un paciente es una razón de estrés. El 42\% (10) de los profesionales revelo que tomar una decisión sobre un paciente cuando el médico no está disponible predispone a que exista presencia de estrés laboral.

Conflicto con otras enfermeras

Para el 13\% (3) de la población encuestada, tener problemas con los superiores es frecuentemente causa de estrés. Otra situación como recibir críticas de un superior, suelen ser frecuentemente fuente de estrés para el 25\% (6) de los participantes.

Otros Factor relacionado al estrés laboral

En la actualidad con la presencia de la PANDEMIA mundial de COVID 19, ha afectado notablemente en el aumento de estrés a los participantes de presente estudio.

\section{DISCUSIÓN}

El nivel de estrés en la población estudiada correspondió al 21\% en un alto nivel de estrés, mientras que el 79\% están expuestos a un bajo nivel de estrés laboral.

Al determinar cuál es el nivel de exposición de estrés por género, se reflejó como resultado que el género femenino es el más expuesto a altos niveles de estrés laboral. Otros autores también describen una relación similar con el género femenino, que se explica desde la formación psicológica de la mujer, las necesidades afectivas, su mecanismo de afrontamiento, el alto nivel de carga laboral (Molina-Jiménez, 2020). 
En el factor relacionado con el ambiente físico (carga de trabajo), el 71\% de los profesionales encuestados mencionan no tener suficiente tiempo para realizar todas las tareas de enfermería, como se menciona en un estudio de una investigación cualitativa realizadas por profesionales de Universidades de Colombia donde se menciona como resultado que para la enfermera la carga del servicio es asumir la responsabilidad sobre todo lo que allí suceda, y realizar acciones que no son de su competencia disciplinar, que es el cuidado integral de enfermería. Tienen que cargar con: culpas, problemas, interacciones, indicadores y protocolos y además el tiempo no les alcanza (Lopera Betancur, 2016).

En el segundo factor el relacionado con el ambiente psicológico (muerte y sufrimiento), el ítems como mayor puntaje fue el de estar presente en la muerte de un paciente el cual genero estrés laboral en un $71 \%$ de los profesionales encuestados; similar a lo expuesto en un estudio realizado en Argentina con enfermeras de unidades de cuidados intensivos, el cual también menciona que las enfermas que presenciaron una escena de muerte en las área criticas fue factor desencadenante en el aumento del estrés laboral (Popp, 2008).

$\mathrm{Y}$ el último factor el relacionado con el ambiente social (conflicto con médicos), el ítem con mayor puntaje fue el de tomar una decisión sobre un paciente cuando el médico no está disponible en un $42 \%$ de los profesionales encuestados predispone a que exista presencia de estrés laboral. Así mismo lo mencionado un estudio realizado en un trabajo de Máster de la Universidad de Miguel Hernández el cual menciona que tomar una decisión no correspondida puede provocar un aumento de estrés Laboral en el incumplimiento de una profesión (Izquierdo Jerez, 2017).

Esta investigación tuvo como fortaleza haber abarcado el total de la población de enfermeros (as) de las dos instituciones objeto de estudio, permitiendo determinar los factores asociados al estrés laboral en este grupo poblacional.

La mayor limitación que se presentó en el desarrollo de la investigación fue al momento de la recolección de encuestas, teniendo en cuenta que el personal labora en turnos rotativos, generando demoras, además de las múltiples ocupaciones del personal que en ocasiones retrasaba la recolección de los datos.

\section{CONCLUSIONES}

El presente artículo acerca del estrés laboral en el personal de Enfermería del área de UCI durante la pandemia de COVID 19 realizado en el Hospital General Dr. Rafael Rodríguez Zambrano de Manta forma parte de la cátedra de Redacción Científica, la cual impulsa a estudiantes universitarios a incursionar en el ámbito de la investigación y desarrollo de artículos científicos con el fin de actualizar y mejorar conocimientos.

El nivel de exposición alto de estrés se evidenció en un 21\% de la población sujeta a este estudio. Los factores con mayor nivel de estrés fueron los relacionados con la "carga laboral” y el de "muerte y sufrimiento", los cuales ambos en uno de sus ítems obtuvieron un alto nivel de estrés laboral superior al 50\% de la población encuestada con actividades como son falta de tiempo en la realización de sus tareas profesionales y la de ver morir a un paciente hospitalizado.

Seguidamente con el factor "conflicto con médicos”, perteneciente al ambiente social la situación que se caracterizó como causa frecuente de estrés corresponde a tomar decisiones sobre un paciente cuando el médico no está disponible.

48 UNESUM-Ciencias. Publicación cuatrimestral. Vol. 4, No. 3 (Número Especial), Año 2020. 
En la investigación, está claro que existe una creciente necesidad de instrumentar a las enfermeras para evaluar los factores estresantes en función de los mecanismos de afrontamiento disponibles, a fin de minimizar la probabilidad de que las personas experimenten estrés.

El estrés tiene diferentes efectos en la vida, afecta tanto en lo físico y en lo emocional, a su vez en otras veces afecta en otras áreas como las de las relaciones interpersonales, desempeño laboral, académico y vida familiar.

\section{REFERENCIAS BIBLIOGRÁFICAS}

Aray Mendoza, D., \& Acosta Andrade, W. (2016). Diseño de un plan de prevención y control de estrés para mejorar el clima laboral del área de mantenimiento de la Facultad de Ciencias Administrativa. Universidad de Guayaquil Facultad de Ciencias Administrativas, Guayaquil. Obtenido de http://repositorio.ug.edu.ec/bitstream/redug/11199/1/TESIS\%20ESTRES\%20LABORAL.pdf

Chiang Vega, M. M., Riquelme Neira, G. R., \& Rivas Escobar, P. A. (Diciembre de 2018). Relación entre Satisfacción Laboral, Estrés Laboral y sus Resultados en Trabajadores de una Institución de Beneficencia de la Provincia de Concepción. Scielo, xx. Obtenido de http://dx.doi.org/10.4067/S0718-24492018000300178

Félix Verduzco, R. O., García Hernández, C., \& Mercado Ibarra, S. M. (2018). EL ESTRÉS EN EL ENTORNO LABORAL. Dialnet, 31-42. Obtenido de https://dialnet.unirioja.es/descarga/articulo/7188504.pdf

Izquierdo Jerez, O. (2017). Estrés y Síndrome de Burnout en profsionales de enfermería. Universidad Miguel Hernandez.

Lopera Betancur, M. A. (2016). El quehacer cotidiano de la enfermera significa soportar la carga. . Revista Cuidarte, 7(2), $\quad$ 1262-1270, $\quad$ http://www.scielo.org.co/scielo.php?script=sci_arttext\&pid=S221609732016000200003\&lng=en\&nrm=iso\&tlng=es.

Lopes Guerrer, F., \& Ferraz Bianchi, E. (Abril de 2015). Estrés de los enfermeros de UCI en Brasil. Scielo. Obtenido de http://scielo.isciii.es/scielo.php?script=sci_arttext\&pid=S1695-61412011000200004

Medina Espinoza, A. L. (2017). Factores asociados al estrés laboral en el personal de enfermeria del area de emergencia y unidad de cuidados intensivos de un hospital de la ciudad de Guayaquil. UNIVERSIDAD CATOLICA DE SANTIAGO DE GUAYAQUIL, Guayaquil. Obtenido de http://repositorio.ucsg.edu.ec/bitstream/3317/8987/1/T-UCSG-PRE-MED-ENF-392.pdf

Molina-Jiménez, T. G.-G.-D. (2020). ESTRÉS PSICOSOCIAL: ALGUNOS ASPECTOS CLÍNICOS Y EXPERIMENTALES. . Obtenido de https://revistas.um.es/analesps/article/view/42951

Ospina Stepanian, A. (2016). SINTOMAS, NIVELES DE ESTRES Y ESTRATEGIAS DE AFRONTAMIENTO EN UNA MUESTRA DE ESTUDIANTES MASCULINOS Y FEMENINOS DE UNA INSTITUCION MILITAR. UNIVERSIDAD CATÓLICA DE COLOMBIA, Bogota. Obtenido de https://repository.ucatolica.edu.co/bitstream/10983/3161/4/TESIS\%20DE\%20MAESTR\%C3\%8DA\%20\%20 Andr\%C3\%A9s\%20Ospina\%20Stepanian.pdf

Popp, M. S. (2008). Estudio preliminar sobre el síndrome de Burnout y estrategias de afrontamiento en enfermeras de unidades de terapia intensiva (UTI). Interdisciplinaria, https://www.redalyc.org/pdf/817/81730850005.pdf.

Reyes , v., \& Milagros, S. (2019). Estrés y calidad de cuidado de la enfermeraen el paciente Hospital Víctor Ramos Guardia Huaraz. UNIVERSIDAD NACIONAL DE TRUJILLO FACULTAD DE ENFERMERIA, Trujillo. Obtenido de https://1library.co/document/4zp8wk7y-estres-calidad-cuidado-enfermera-paciente-hospitalvictor-guardia.html\#fulltext-content

Sarsosa Prowesk, K., \& Charria Ortiz, V. H. (Abril de 2018). Estrés laboral en personal asistencial de cuatro instituciones de salud nivel III de Cali, Colombia. Scielo. Obtenido de http://dx.doi.org/10.22267/rus.182001.108 
Vargas García, B., \& Garcia Alcivar, R. (2018). EL ESTRÉS LABORAL Y SU INCIDENCIA EN LOS TRABAJADORES. ResearchGate. https://www.researchgate.net/publication/328781994_El_estres_laboral_y_su_incidencia_en_el_desempeno_ de_los_trabajadores

Vicente, M., \& Yesenia, J. (2017). EL ESTRES LABORAL Y SU INFLUENCIA EN EL CLIMA ORGANIZACIONAL. UNIVERSIDAD NACIONAL JOSÉ FAUSTINO SÁNCHEZ, HUACHO. Obtenido de http://repositorio.unjfsc.edu.pe/bitstream/handle/UNJFSC/1856/TFCE-0311.pdf?sequence=1\&isAllowed=y\#: :text=El\%20estr\%C3\%A9s\%20laboral\%20aparece\%20cuando,y\%20me ntal\%2C\%20como\%20consecuencia\%20hay

Yunda Ibarra, A. P. (2018). Reducción del estrés laboral mediante la aplicación de la Técnica de Relajación. http://www.dspace.uce.edu.ec/bitstream/25000/15343/1/T-UCE-0020-TO033-2018.pdf 